\title{
A Hybrid-Binarization Approach for Degraded Document Enhancement
}

\author{
Moushumi Zaman Bonny ${ }^{1,2}$, Mohammad Shorif Uddin ${ }^{1}$ \\ ${ }^{1}$ Department of Computer Science and Engineering, Jahangirnagar University, Savar, Dhaka, Bangladesh \\ ${ }^{2}$ Department of Computer Science and Engineering, Daffodil International University, Dhaka, Bangladesh \\ Email: zaman.moushumi@gmail.com,bonny.cse@diu.edu.com
}

How to cite this paper: Bonny, M.Z. and Uddin, M.S. (2020) A Hybrid-Binarization Approach for Degraded Document Enhancement. Journal of Computer and Communications, 8, 1-11. https://doi.org/10.4236/jcc.2020.812001

Received: September 30, 2020 Accepted: November 28, 2020 Published: December 1, 2020

Copyright () 2020 by author(s) and Scientific Research Publishing Inc. This work is licensed under the Creative Commons Attribution International License (CC BY 4.0).

http://creativecommons.org/licenses/by/4.0/

(c) (i) Open Access

\begin{abstract}
Images get degraded because of unbalanced enlightenment including text-smearing, ink-bleeding, degradation of ink over time, manuscript characters from background coming out and blended with the characters of the main side etc. So, degraded-document enhancement is a challenging issue. In recent years, several binarization approaches are proposed to enhance these images. These techniques have focused on finding a suitable global threshold value or a local threshold value for every region to eliminate the degradations. A hybrid approach can be a good solution to deal with all these matters together. This paper proposes a hybrid approach of binarization for degraded documents to produce better quality result. Then, the performance of the proposed technique is evaluated using DIBCO 2010 to DIBCO 2018 databases and compared with the existing methods which confirmed that the proposed method is robust, efficient. Finally, a direction towards future works and challenges is stated.
\end{abstract}

\section{Keywords}

Degradation, Enhancement, Hybridization, Image Manuscript, Thresholding, Binary Image

\section{Introduction}

There are numerous old and historical documents and manuscripts which are stored in libraries and archives. These documents have essential information about the heritage of human. Different ecological issues, inappropriate management and bad-quality materials of the paper degraded these documents [1] [2] [3]. So, the analysis on the degradation of manuscripts is a challenging area for researchers nowadays. 
Binarization is the primary step of analyzing documents. It is a technique of conversion from grayscale or into a binary image. The consequent steps are vastly reliant on the accomplishment of binarization and the success of binarization can affect the preservation of the significant information of the degraded documents.

There are different types of binarization approaches which contain several advantages and drawbacks. So, it is very demandable to develop a quick, ideal and appropriate method for every degraded document [4].

The core principle of this research is to offer an image binarization technique to satisfy different purposes. It attempts to unite the benefits of local and global thresholding.

Our paper is structured as follows: In Section 2 we have illustrated literature review. Section 3 presented our proposed binarization technique briefly. In Section 4 , the performance evaluation is briefly described. We have discussed the advantages of the proposed method in Section 5 and conversed about the future works and challenges in Section 6. Finally, in Section 7, we have concluded the paper along with some future research directions.

\section{Literature Review}

In recent years, many researchers worked on binarization techniques. A complete evaluation on image binarization along with some noble and benchmarked binarization methods are presented below:

Lu et al. [4] presented a contrast-enhancement based thresholding technique. They focused on the difference of the contrast of grayscale image in different regions of input image.

Mollah et al. [5] presented a binarization method for the images of business cards. Based on intensity-variance, a card image is separated into small blocks which can be extracted from background. The areas without text are removed and the texts are exacted and thresholded by applying a local adaptive method.

Darek Bradley et al. [6] proposed a vigorous and simple binarization technique which depends on spatial variation in illumination.

Badekas et al. [7] presented a binarization technique for color pictures where texts are mixed with the background.

Otsu [8] presented a global thresholding technique. It can convert grayscale images to bi-level images and can divide the pixels into background and foreground divisions. The Otsu's technique works well with the images with bi-modal model.

Sauvola [9] presented another procedure which uses standard deviation to find out the threshold value. It is an upgraded version of Niblack's technique.

Nick et al. [10] presented local thresholding technique. In this process, the value of thresholding is moved downward to eliminate noise and to lessen the contrast problem.

Shafait et al. [11] proposed local adaptive thresholding technique. Local adap- 
tive thresholding is used for local binarization. A window of size $\mathrm{N} \times \mathrm{N}$ moves over the whole picture and a thresholding value is calculated for every local region. This technique provides ideal outcome with the images affected by low resolutions, blurring and non-uniform illumination etc.

However, different researchers presented different methods for the enhancement of degraded documents. But all these methods have some benefits along with some drawbacks. So, a vigorous binarization technique which is applicable on any degraded document for the enhancement is still in high demand to develop.

Through these observations, in this paper, we have presented a hybrid technique with the desire of better output with proper enhancement. Our technique presents a robust binarization approach where we hybridized some thresholding methods to get better result than any other approach.

\section{Proposed Methodology}

The binarization method is used as the main part in different analyses of documents. On the other side, the method must be simple and suitable for every document analysis. Figure 1 shows the flowchart of the steps of the proposed hybrid-binarization technique.

The steps of the proposed binarization technique are detailed and explained below:

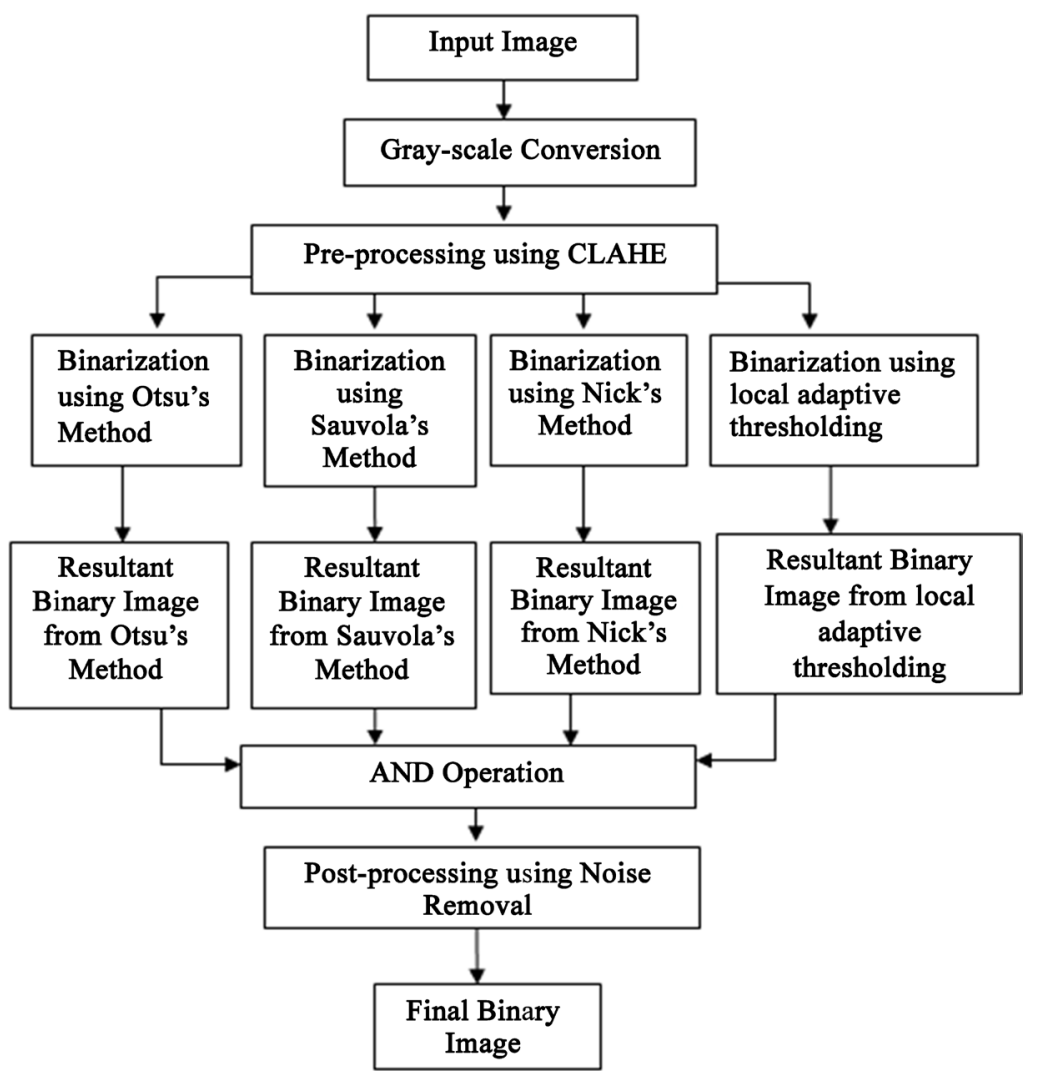

Figure 1. Proposed hybrid-binarization technique. 


\subsection{Inputting Image}

Inputting image is the initial step of this procedure. Images are captured by camera or obtained by using secondary sources such as scanner.

\subsection{Converting to Gray-Scale Image}

After completing the above step, we need to verify the image to know the format of the image. When the inputted image is in RGB format then the image will be transferred into grayscale format. But, if the image is in grayscale, then it will be preprocessed in the next stage.

\subsection{Preprocessing}

The preprocessing step is the next stage for the grayscale image. Generally, preprocessing is required to eliminate the imperfections of the input-image. This step facilitates the hybridization step. The contrast of the grayscale-image is made better and enhanced by applying CLAHE (Contrast Limited Adaptive Histogram Equalization). In each segment of the image, it improves the local contrast and restricts the amplification of noise.

\subsection{Hybrid-Binarization}

After grayscale enhancement, we go through a hybrid thresholding technique by uniting four ideal methods: Otsu's technique, Sauvola's technique, Nick's method and local adaptive thresholding technique.

Otsu's technique performs best for the images with apparent bi-modal model and Sauvola's technique makes enhanced outcome with least amount of noise and but it is not able to differentiate text with very low-contrast and illuminated-texture.

Local adaptive technique gives perfect result for the images which are affected by low-resolution, shading, and blurring. Nick's method performs very well in dealing with noise. So, considering the advantages of these methods, we combined these techniques for binarization to get better performance.

\subsection{Post-Processing}

The binary image will go through post-processing. We have done noise removal operation for the enhancement of the document.

\section{Experimental Results}

For evaluating the performance of our technique, we have done some simulations using the datasets of DIBCO-2010 to DIBCO 2018 which include handwritten and printed documents. In addition, the proposed method is compared with some standard binarization techniques, such as Otsu's, Savoula's, Nick's, and local adaptive thresholding methods on the basis of accuracy rate and computation time.

In Figure 2(a), the inputted image is taken from DIBCO-2010 database, in 
Figure 2(b), we presented the resultant image produced from Otsu's method, in Figure 2(c) we depicted output from the Sauvola's method, in Figure 2(d), we have shown the outcome of Nick's technique, in Figure 2(e), we demonstrated the result of local adaptive thresholding method and in Figure 2(f), we depicted the result of proposed method.

We have completed similar actions in Figures 3-9 for the images collected from DIBCO-2011 DIBCO-2012 DIBCO-2013, DIBCO-2014, DIBCO-2016, and DIBCO-2017 and DIBCO-2018 datasets respectively.

We have also publicized the accuracy rate and computation time for the

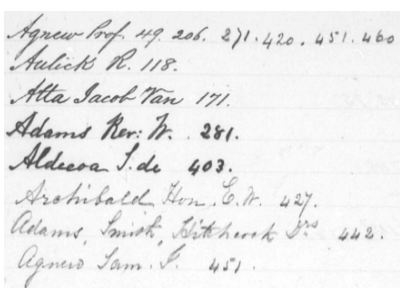

(a)

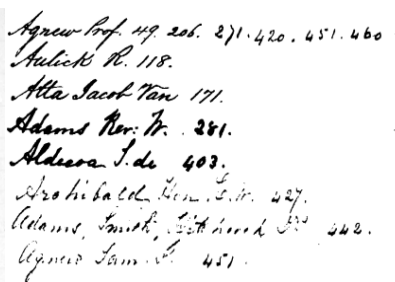

(b)

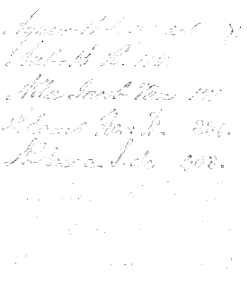

(c)

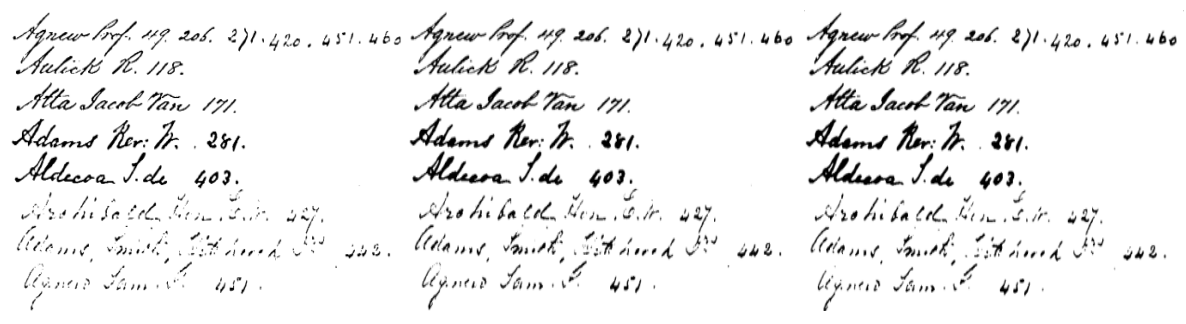

(d) (e) (f)

Figure 2. (a) Inputted image (DIBCO 2010); (b) Result of Otsu technique; (c) Result of Sauvola's technique; (d) Result of Nick's technique; (e) Result of local adaptive thresholding; (f) Result of proposed technique.

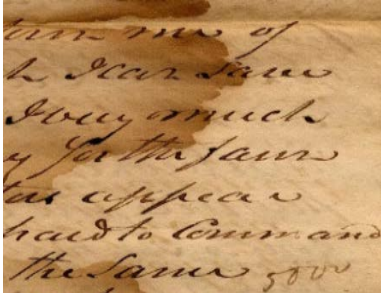

(a)

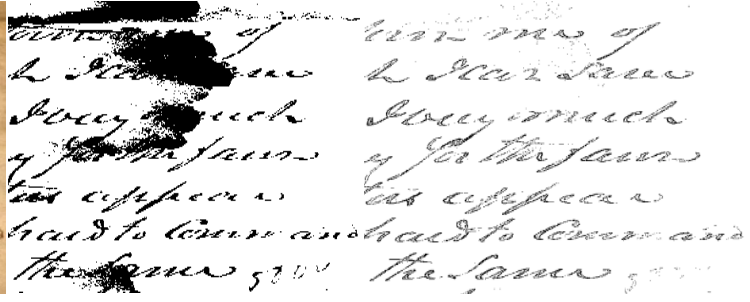

(b) (c)

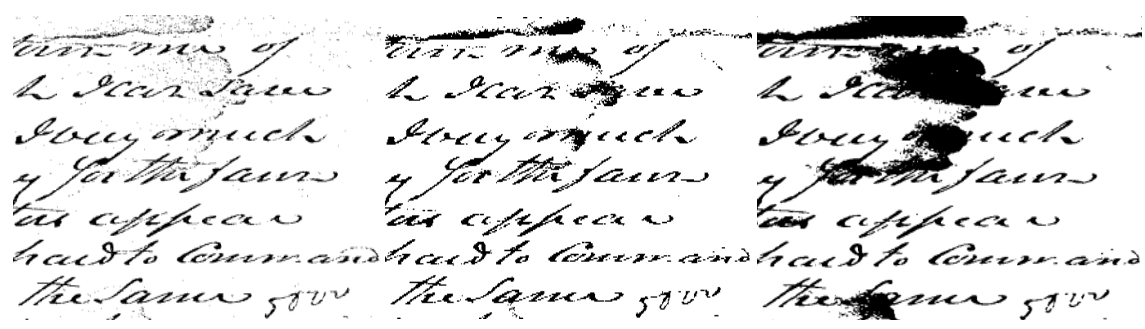

(d)

(e)

(f)

Figure 3. (a) Inputted image (DIBCO 2011); (b) Result of Otsu technique; (c) Result of Sauvola's technique; (d) Result of Nick's technique; (e) Result of local adaptive thresholding; (f) Result of proposed technique. 


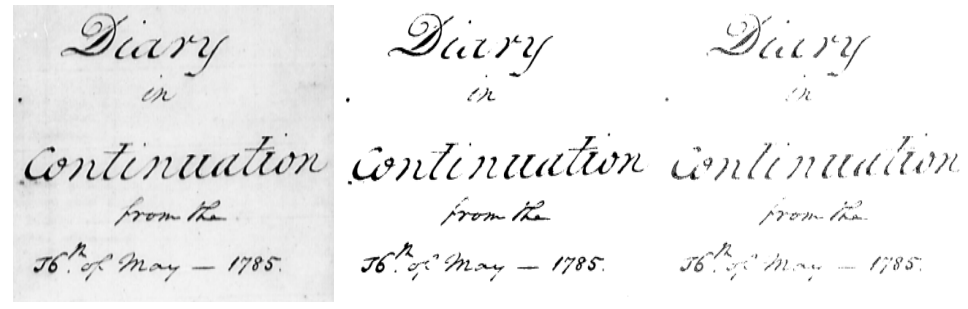

(a)

(b)

(c)

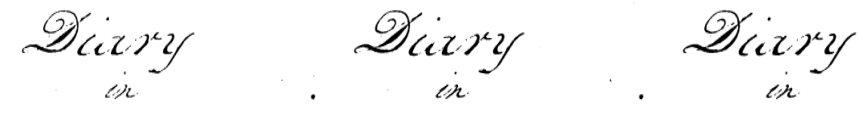

continuation continuation continuation

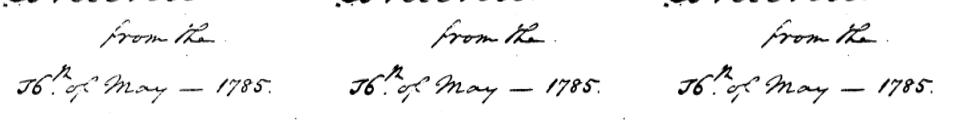

(d)

(e)

(f)

Figure 4. (a) Inputted image (DIBCO 2012); (b) Result of Otsu technique; (c) Result of Sauvola's technique; (d) Result of Nick's technique; (e) Result of local adaptive thresholding; (f) Result of proposed technique.

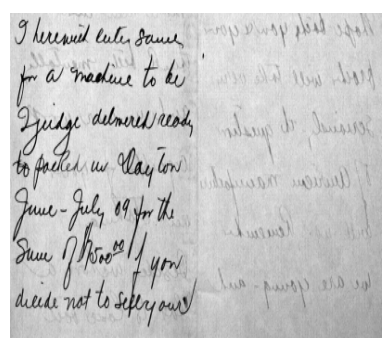

(a)

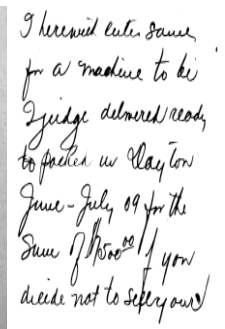

(b)

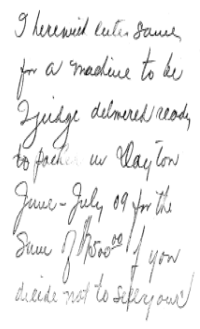

(c)

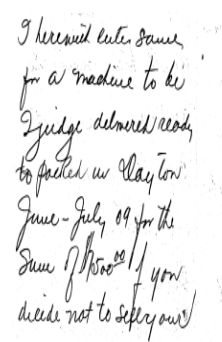

(d)

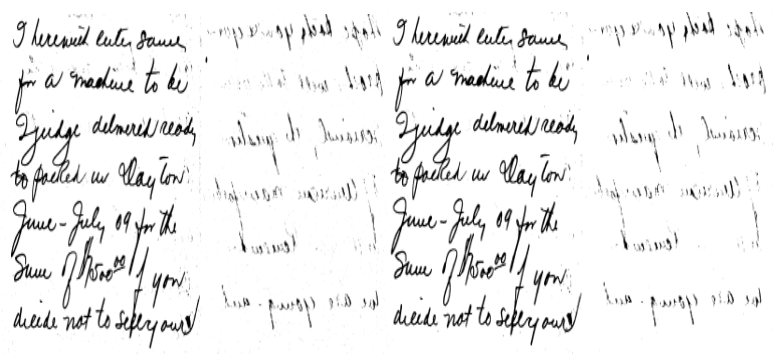

(e)

(f)

Figure 5. (a) Inputted image (DIBCO 2013); (b) Result of Otsu technique; (c) Result of Sauvola's technique; (d) Result of Nick's technique; (e) Result of local adaptive thresholding; (f) Result of proposed technique.

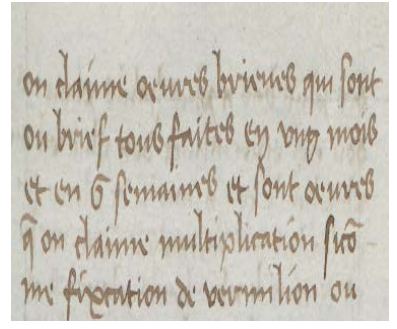

(a)

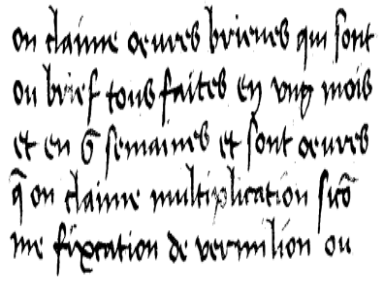

(b)

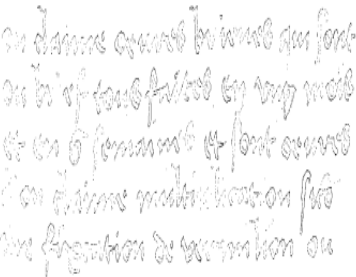

(c) 


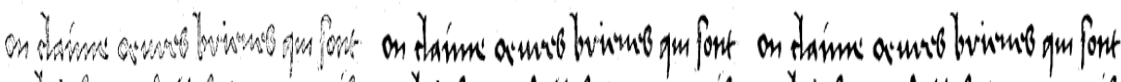

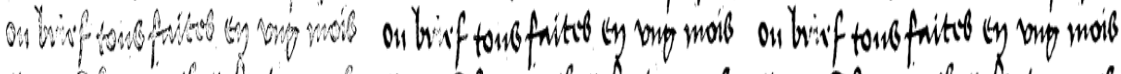

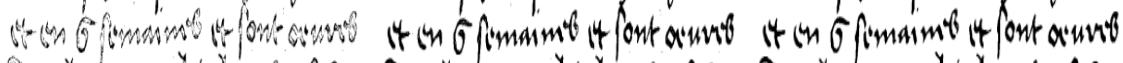

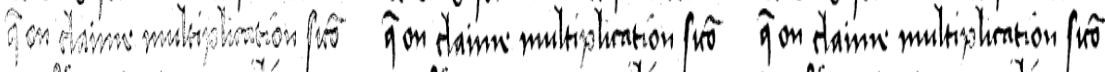

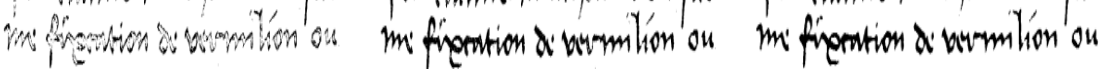

(d)

(f)

Figure 6. (a) Inputted Image (DIBCO 2014); (b) Result of Otsu technique; (c) Result of Sauvola's technique; (d) Result of Nick's technique; (e) Result of local adaptive thresholding; (f) Result of proposed technique.

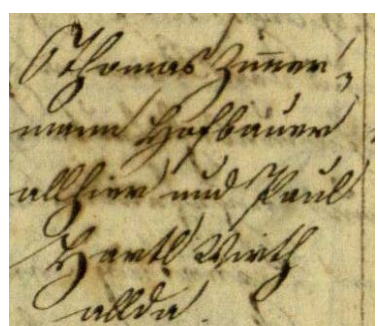

(a)

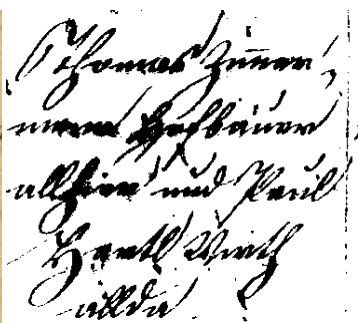

(b)

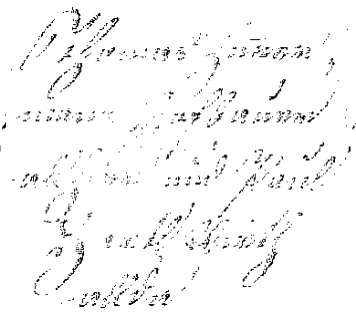

(c)

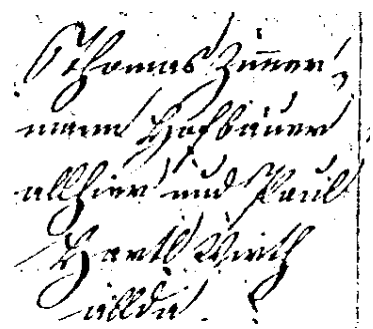

(d)

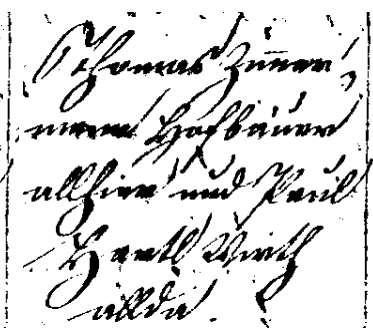

(e)

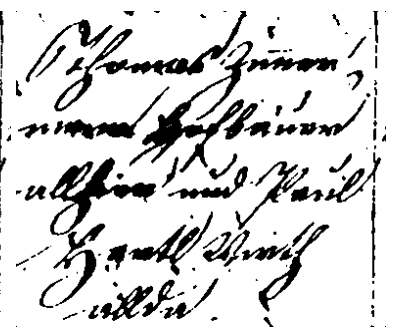

(f)

Figure 7. (a) Inputted image (DIBCO 2016); (b) Result of Otsu technique; (c) Result of Sauvola's technique; (d) Result of Nick's technique; (e) Result of local adaptive thresholding; (f) Result of proposed technique.

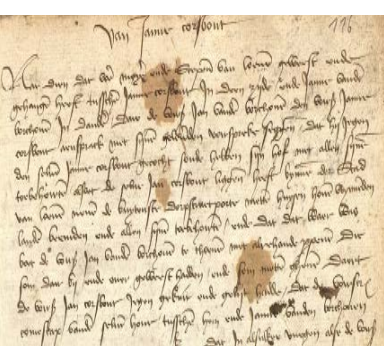

(a)

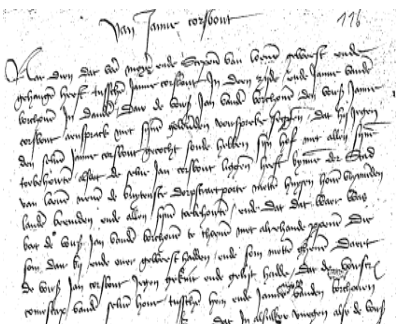

(d)

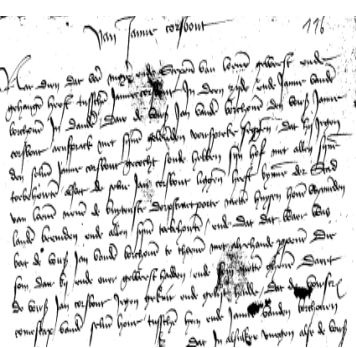

(b)

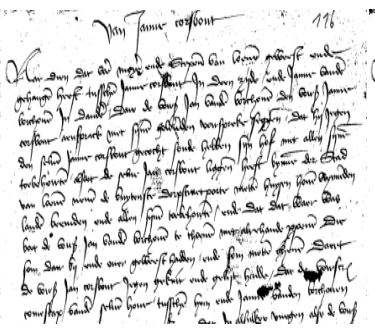

(e)

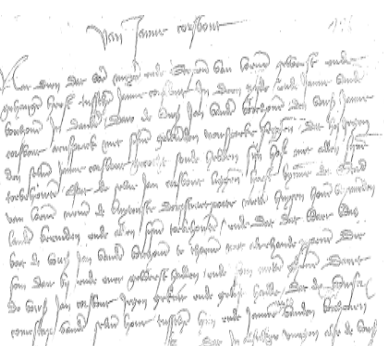

(c)

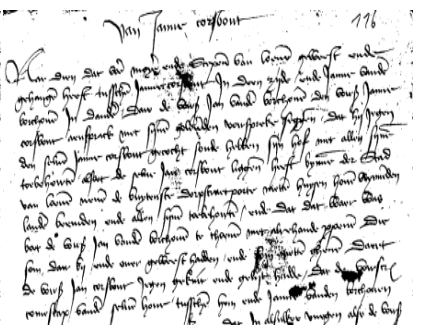

(f) 
Figure 8. (a) Inputted image (DIBCO 2017); (b) Result of Otsu technique; (c) Result of Sauvola's technique; (d) Result of Nick's technique; (e) Result of local adaptive thresholding; (f) Result of proposed technique.

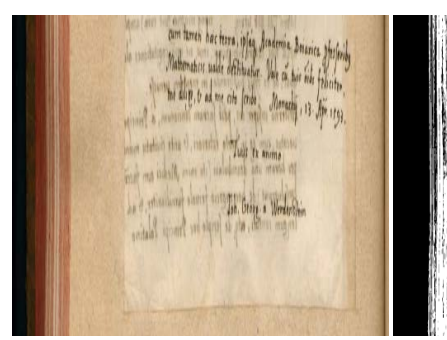

(a)

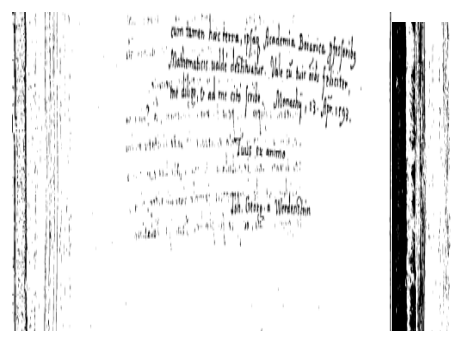

(d)

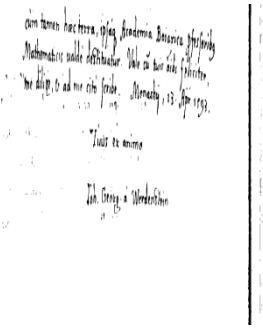

(b)

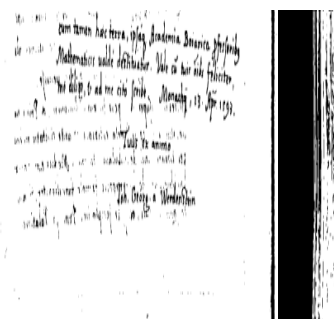

(e)

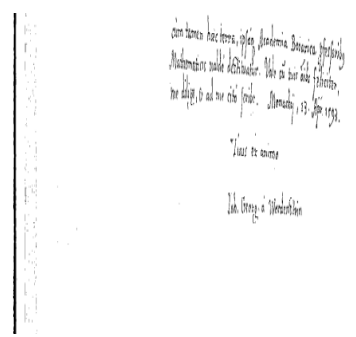

(c)

Figure 9. (a) Inputted image (DIBCO 2018); (b) Result of Otsu technique; (c) Result of Sauvola's technique; (d) Result of Nick's technique; (e) Result of local adaptive thresholding; (f) Result of proposed method.

evaluation of the results in Table 1 . We have applied the following method to compute the accuracy A:

$$
\mathrm{A}=1-\operatorname{sum} \operatorname{sum}\left(\left|\left(I M_{1}-I M_{2}\right)\right|\right) / \operatorname{sum}\left(\operatorname{sum}\left(I M_{1}\right)\right)
$$

In Equation (1) [12], $I M_{1}$ is the original image and $I M_{2}$ is the output generated from proposed technique.

Table 1. Performance evaluation of the proposed technique along with different binarization techniques.

\begin{tabular}{cccc}
\hline Datasets & Binarization Methods & Accuracy rate (\%) & Time (sec) \\
\hline DIBCO 2010 & Otsu & 99.41 & 1.87 \\
& Local adaptive & 99.33 & 1.11 \\
Sauvola & 92.84 & 0.68 \\
Nick & 97.51 & 0.65 \\
H-DIBCO 2011 & Oroposed method & 99.55 & 2.70 \\
& Otsu & 91.96 & 1.12 \\
& Local adaptive & 82.40 & 0.95 \\
& Sauvola & 74.15 & 1.06 \\
& Nick & 78.84 & 1.28 \\
& Proposed method & 92.22 & 1.78 \\
\hline
\end{tabular}




\section{Continued}

\begin{tabular}{|c|c|c|c|}
\hline \multirow[t]{5}{*}{ H-DIBCO 2012} & Otsu & 99.96 & 1.24 \\
\hline & Local adaptive & 99.25 & 1.96 \\
\hline & Sauvola & 98.08 & 1.18 \\
\hline & Nick & $99 . .43$ & 1.57 \\
\hline & Proposed method & 99.37 & 2.62 \\
\hline \multirow[t]{5}{*}{ DIBCO 2013} & Otsu & 99.26 & 1.96 \\
\hline & Local adaptive & 98.16 & 1.55 \\
\hline & Sauvola & 97.76 & 1.18 \\
\hline & Nick & 99.11 & 1.85 \\
\hline & Proposed method & 99.65 & 6.95 \\
\hline \multirow[t]{5}{*}{ DIBCO 2014} & Otsu & 98.72 & 1.25 \\
\hline & Local adaptive & 98.68 & 1.11 \\
\hline & Sauvola & 86.66 & 1.16 \\
\hline & Nick & 92.52 & 1.14 \\
\hline & Proposed method & 98.82 & 2.10 \\
\hline \multirow[t]{5}{*}{ DIBCO 2016} & Otsu & 95.64 & 1.10 \\
\hline & Local adaptive & 93.36 & 0.92 \\
\hline & Sauvola & 77.89 & 0.85 \\
\hline & Nick & 89.10 & 1.04 \\
\hline & Proposed method & 95.69 & 1.48 \\
\hline \multirow[t]{5}{*}{ DIBCO 2017} & Otsu & 98.24 & 1.25 \\
\hline & Local adaptive & 98.43 & 1.21 \\
\hline & Sauvola & 89.80 & 1.60 \\
\hline & Nick & 96.63 & 1.64 \\
\hline & Proposed method & 98.68 & 4.37 \\
\hline \multirow[t]{5}{*}{ DIBCO 2018} & Otsu & 99.43 & 0.79 \\
\hline & Local adaptive & 92.08 & 1.15 \\
\hline & Sauvola & 85.50 & 0.82 \\
\hline & Nick & 87.05 & 0.85 \\
\hline & Proposed method & 97.59 & 2.09 \\
\hline
\end{tabular}

\section{Discussions}

Therefore, the outputs show the robustness of our proposed technique. It is found that, our proposed method and Otsu's method have shown enhanced performance comparing to the other techniques because these two have shown utmost accuracy rate. The local adaptive thresholding gave somewhat good outputs. But, rests of the techniques publicized the comparatively lesser performance to these techniques. 


\section{Future Works and Challenges}

We have presented a technique that combines the advantages of different binarization techniques such as global and local binarization. So, future researchers can focus on parameter tuning, noise removing, post-processing for qualitative binarization. The post-processing can reduce the performance by increasing the execution time but it can develop the quality of the manuscripts. Several filters could be used for noise problems and these filters could reduce black spots from the final image. In this way, we can preserve the important information of the degraded documents.

\section{Conclusion}

Historical documents hold important information of ancient times. High-quality techniques of binarization can recover the texts from the degraded document image. The earlier studies show that there is no efficient technique of binarization for diverse types of degraded document images. A variety of binarizations are used to enhance these degraded documents. So it is necessary to propose a quick and ideal technique of binarization which is appropriate for all kinds of degraded documents. We proposed a hybrid binarization approach to produce good quality image and attempted to merge the benefits of local and global thresholding methods. The experimentations over the datasets DIBCO 2010 to DIBCO 2018 confirmed that the performance of the proposed technique was superior in terms of the accuracy rate.

\section{Conflicts of Interest}

The authors declare no conflicts of interest regarding the publication of this paper.

\section{References}

[1] Stathis, P., Kavallieratou, E. and Papamarkos, N. (2008) An Evaluation Technique for Binarization Algorithms. Journal of Universal Computer Science, 14, 3011-3030. https://doi.org/10.1109/ICPR.2008.4761546

[2] Mehmet, S. and Sankur, B. (2004) Survey over Image Thresholding Techniques and quantitative Performance Evaluation. Journal of Electronic Imaging, 13, 146-165. https://doi.org/10.1117/1.1631315

[3] Gaceb, D., Lebourgeois, F. and Duong, J. (2013) Adaptative Smart-Binarization Method: For Images of Business Documents. 2013 12th International Conference on Document Analysis and Recognition, Washington DC, 25-28 August 2013. https://doi.org/10.1109/ICDAR.2013.31

[4] Lu, D., Huang, X., Liu, C., Lin, X., Zhang, H. and Yan, J. (2016) Binarization of Degraded Document Image Based on Contrast Enhancement. 2016 35th Chinese Control Conference (CCC), Chengdu, 27-29 July 2016, 4894-4899. https://doi.org/10.1109/ChiCC.2016.7554113

[5] Mollah, A.F., Basu, S., Das, N. and Sarkar, R. (2009) Binarizing Business Card Images for Mobile Devices. International Conference on Advances in Computer Vi- 
sion and IT, Aurangabad, 16-19 December 2009, 968-975.

[6] Bradley, D. and Roth, G. (2011) Adaptive Thresholding Using Integral Image. Journal of Graphics Tools, 12, 13-21.

https://doi.org/10.1080/2151237X.2007.10129236

[7] Badekas, E. and Papamarkos, N. (2007) Optimal Combination of Document Binarization Techniques Using a Self-Organizing Map Neural Network. Engineering Applications of Artificial Intelligence, 20, 11-24. https://doi.org/10.1016/j.engappai.2006.04.003

[8] Otsu, N. (1979) A Thresholding Selection Method from Gray-Scale Histogram. IEEE Transactions on System, Man, and Cybernetics, 9, 62-66.

https://doi.org/10.1109/TSMC.1979.4310076

[9] Sauvola, J. and Pietikäinen, M. (2000) Adaptive Document Image Binarization. Pattern Recognition Society, 33, 225-236. https://doi.org/10.1016/S0031-3203(99)00055-2

[10] Khurshid, K., Siddiqi, I., Faure, C. and Vincent, N. (2009) Comparison of Niblack Inspired Binarization Methods for Ancient Documents. Document Recognition and Retrieval XVI, 7247, 72470U. https://doi.org/10.1117/12.805827

[11] Shafait, F., Keysers, D. and Breuel, T. (2008) Efficient Implementation of Local Adaptive Thresholding Techniques Using Integral Images. Document Recognition and Retrieval XV, 6815, 681510. https://doi.org/10.1117/12.767755

[12] Image Error Measurements (2010). https://www.mathworks.com/matlabcentral/fileexchange/29500-image-error-measu rements 